\title{
Numerical Simulation of Acoustic Wave Propagating in a Spherical Object Filled with Insulating Oil
}

\author{
D. Wotzka, D. ZMARZEY* AND T. Boczar
}

Faculty of Electrical Engineering, Automatic Control and Informatics, Opole University of Technology

Mikołajczyka 5, 45-271 Opole, Poland

Results of a numerical simulation of an acoustic wave that propagates in a spherical object filled with insulating oil are presented in the paper. The sinusoidal acoustic wave is generated by an electro-acoustic transducer mounted in the middle of the object. Presented results depict acoustic pressure distribution within the transient and the steady states in a two-dimensional object. Furthermore, comparison of short time simulations adapting different mesh element size is presented.

PACS numbers: 02.60.Lj

\section{Introduction}

The aim of the scientifically-investigative works performed by authors is definition of a mathematical model of an acoustic emission (AE) source whose acoustic response reflects the real wave generated by a partial discharge appearing in insulating transformer oil [1-7]. Within the scope of the authors' research works a number of numerical simulations have been performed [8-12]. Beginning with simplified models - one of them presented in this paper - sophisticated models are to be created and analyzed. Simulations presented in this paper consider a small glass jar that is filled with insulating oil, inside of which an acoustic wave source (a piezoelectric transducer) is mounted. In the simulations performed we consider time-dependent analysis of the acoustic pressure distribution inside the glass jar. The paper includes short description of the applied software, geometry of the object under study, simulation settings and results of numerical calculations performed.

\section{Software applied for numerical calculations}

For the acoustic pressure propagation estimation the COMSOL Multiphysics application and in particular the acoustic pressure (AP) module has been applied. The AP module includes already definition of the general wave equation and combines it together with boundary conditions into a system of partial differential equations (PDEs) which are then solved by use of the field electron microscopy (FEM) method. The geometry of the object under study is modeled within the CAD tool supported by COMSOL. In general, the object consists of a set of subdomains. Every subdomain is divided into a number of elements during the meshing procedure. In case of time-harmonic wave simulation the mesh element size $\Delta x$ should be a function of the wavelength $\lambda$. The physics on a model is described by subdomain settings:

* corresponding author; e-mail: dzmarzly@gmail.com by coefficients that define the PDE directly or by material properties, by sources and sinks which define the desired physics indirectly (software performs translation of the material properties into PDE coefficients automatically). Considered model assumes definition of the Lagrange finite elements of order two (quadratic elements with polynomial shape function) with one dependent variable that relates to the acoustic pressure $p[\mathrm{~Pa}]$. Detailed information about governing equations can be found in [8-10].

\section{Object geometry and simulation setting}

As already mentioned the object under study depicts a glass jar filled with insulating oil (Fig. 1). The jar dimensions are as follows: radius $R=5 \mathrm{~cm}$, height $H=$ $20 \mathrm{~cm}$. In the center of the glass jar the electro-acoustic transducer is mounted on a copper stick.

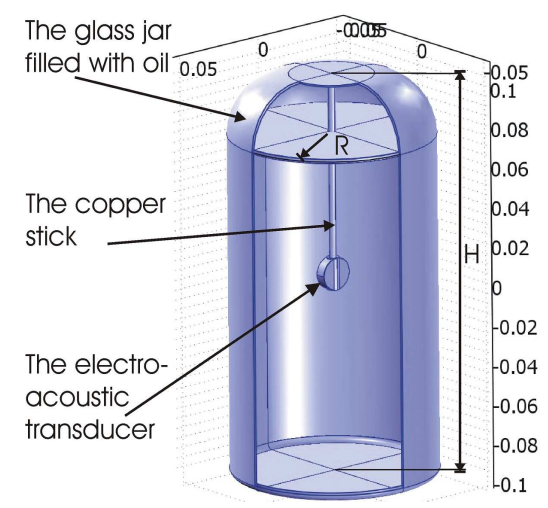

Fig. 1. Object under study depicting glass jar filled with insulating oil.

For proper calculations the appropriate number of mesh elements is essential; it is common to say that there should be at least 20 elements per wavelength. Considering picture presented in Fig. 1, application of this rule produces millions of mesh elements and a number of degrees of freedom (DoF) which is impossible to solve by authors with their equipment. Thus, the model geometry 


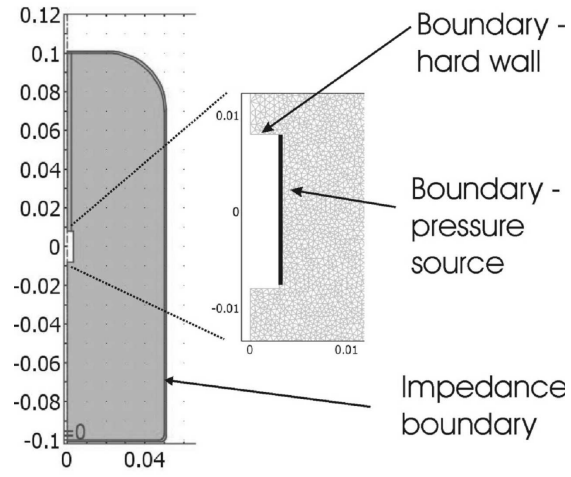

Fig. 2. The part of the object under study applied in the simulations (left), the transducer part emitting sound (right) with boundaries defined.

has been simplified to two dimensions where additionally the symmetry axis has been adopted. The simplified model depicting mesh elements and boundaries modeled is presented in Fig. 2.

The boundary conditions are crucial for the simulation results and are specified as follows:

1. on all jar exterior boundaries the impedance boundary condition is defined: $Z=1624 \times 10^{4}$;

2. on all interior boundaries continuity condition is defined;

3. hard wall boundary at the transducer collateral surface, as marked in Fig. 2;

4. at the boundary between oil and transducer emitting side sinusoidal pressure changes are defined as follows: $p=100 \cos (2 \pi f t)$, where $f=100 \mathrm{kHz}$.

The oil filling the glass jar has the following parameters: density $\rho_{0}=890 \mathrm{~kg} / \mathrm{m}^{3}$, speed of sound $c_{\mathrm{s}}=$
$1390 \mathrm{~m} / \mathrm{s}$. Properties of the glass jar are as follows: density $\rho_{0}=7800 \mathrm{~kg} / \mathrm{m}^{3}$, speed of sound $c_{\mathrm{s}}=5600 \mathrm{~m} / \mathrm{s}$. The initial values modeled are as follows: $p\left(t_{0}\right)=0$ and $\partial p / \partial t\left(t_{0}\right)=0$. Time dependent analysis presented in this paper considers time steps $\Delta t=1 \mu \mathrm{s}$; at frequency $f=100 \mathrm{kHz}$ and wavelength $\lambda=13.9 \mathrm{~mm}$ there follow ten time steps per one generation period.

\section{Influence of the mesh element size on calculation results}

In order to analyze the influence of the mesh element size on calculation results four simulations have been performed each considering ten time steps per one generation period. The cases differ in the mesh element size $\Delta x$ :

1. $\Delta x \leq 0.695 \mathrm{~mm}$, producing 174264 DoF (one period solution time: $18 \mathrm{~h}$ ) - serves as reference value;

2. $\Delta x \leq 1.39 \mathrm{~mm}$, producing $44384 \mathrm{DoF}$ (one period solution time: $12 \mathrm{~h}$ );

3. $\Delta x \leq 2.78 \mathrm{~mm}$, producing $12708 \mathrm{DoF}$ (one period solution time: $5 \mathrm{~h}$ );

4. $\Delta x \leq 6.61 \mathrm{~mm}$, producing $6701 \mathrm{DoF}$ (one period solution time: $1 \mathrm{~h}$ ).

Table shows pressure values for the four mesh cases mentioned above calculated at simulation time $t=10 \mu \mathrm{s}$ at two positions: $(r, z)=(6,0) \mathrm{mm}$ and $(r, z)=$ $(14,0) \mathrm{mm}$, respectively. Assuming the first case taken as reference value $v_{0}$, the percent error $\delta$ has been calculated. $\delta$ is less than $1.3 \%$ if at least five elements per wavelength $\lambda$ are adopted and $\delta$ is about $4 \%$ if just two elements per $\lambda$ are adopted. This leads to a conclusion that it is sufficient to perform simulations where there are five to ten mesh elements for a wavelength adopted.

Comparison of calculations for the four mesh cases.

TABLE

\begin{tabular}{c|c|c|c|c}
\hline \hline Mesh case no. & 1 & 2 & 3 & 4 \\
\hline max elem. size $\Delta x[\mathrm{~mm}]$ & 0.695 & 1.39 & 2.78 & 6.61 \\
no. of elem. per $\lambda$ & 20 & 10 & 5 & 2.1 \\
\hline$p[\mathrm{~Pa}]$ at pos. $(r, z)=(6,0) \mathrm{mm}$ & -77.52 & -77.17 & -77.26 & -74.53 \\
percent error $\delta[\%]$ & $v_{0}$ & 0.4515 & 0.3354 & 3.8571 \\
\hline$p[\mathrm{~Pa}]$ at pos. $(r, z)=(14,0) \mathrm{mm}$ & 45.93 & 46.22 & 46.53 & 43.85 \\
percent error $\delta[\%]$ & $v_{0}$ & 0.6314 & 1.3063 & 4.5286
\end{tabular}

\section{Simulation results for the mesh case 3}

This simulation considers the case where there are ten mesh elements per one wavelength modeled, and an error rate of about $0.5 \%$ is taken into account. Simulation results presented in Figs. 3 and 4 depict acoustic pressure $p[\mathrm{~Pa}]$ as surface plots. In Fig. 3, left, one can recognize 


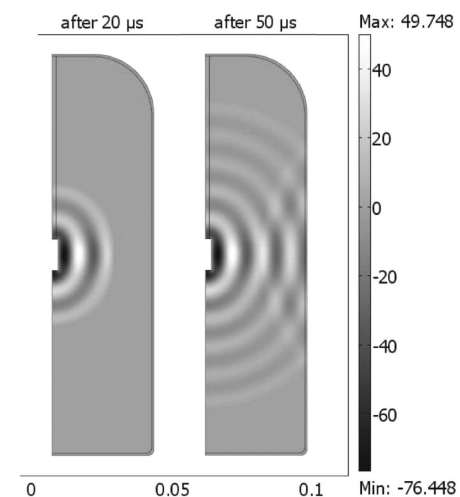

Fig. 3. Surface plots depicting distribution of the acoustic pressure $p[\mathrm{~Pa}]$ at two simulation time steps: $t=20 \mu \mathrm{s}$ and $t=50 \mu \mathrm{s}$.

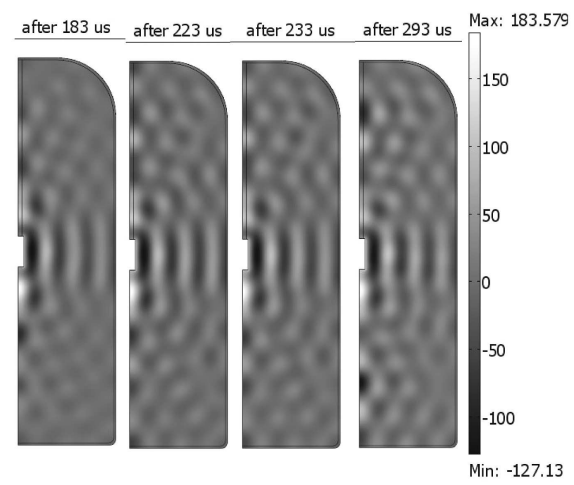

Fig. 4. Surface plots depicting steady state pressure distribution inside the object.

the first and second crests of the acoustic wave in the vicinity of source after $20 \mu \mathrm{s}$ (two periods). In Fig. 3, right, there are first interferences, with the wave reflected on the jar surface, to be recognized after $50 \mu \mathrm{s}$.

In Fig. 4 the four pictures depict acoustic pressure distribution after $18.3,22.3,23.3,29.3$ generation periods respectively. One can recognize that the pressure distribution is the same for all cases. This leads to the conclusion that the steady state has been achieved.

In Fig. 5 the pressure distribution along the line described by coordinate points $x_{1}$ and $x_{2}$, where $x_{1}=$ $(3,0) \mathrm{mm}$ and $x_{2}=(50,0) \mathrm{mm}$, is presented. It is to be recognized that the pressure values do not differ from each other in a significant matter. The differences in values are probably due to the percent error $\delta$ related to the mesh element size.

Figure 6 depicts motion of the acoustic wave. One can recognize that the pressure amplitude $p$ decreases with increasing distance to the sound source.

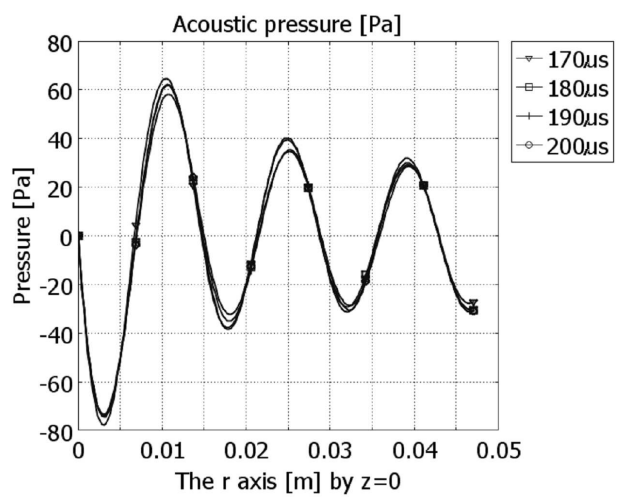

Fig. 5. Line plots depicting steady state pressure distribution along the line described by coordinate points $x_{1}=(3,0) \mathrm{mm}$ and $x_{2}=(50,0) \mathrm{mm}$.

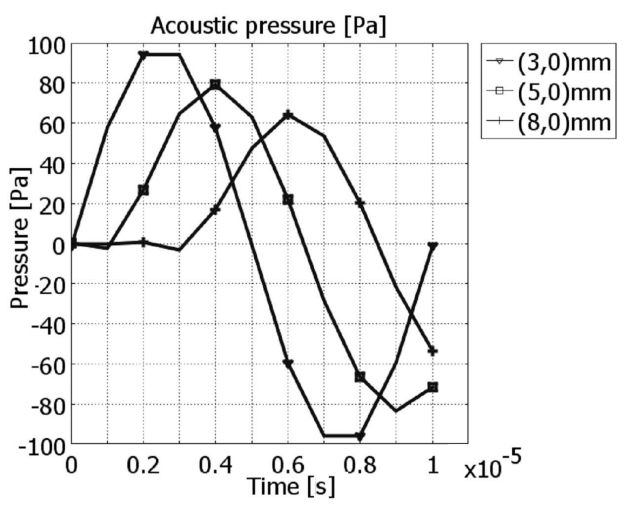

Fig. 6. Time runs of the acoustic pressure $p[\mathrm{~Pa}]$ at three coordinate points $(r, z) \mathrm{mm}$.

\section{Summary}

Paper presented simulation of a sinusoidal acoustic wave inside a glass jar filled with insulating oil. Distribution of the acoustic pressure has been calculated numerically by use of the pressure acoustic module provided with the COMSOL Multiphysics software. The sound wave was initiated by a piezoelectric transducer mounted in the center of the glass jar. A comparison of short time simulations in a two-dimensional object adopting different mesh element size was performed. Further, a long time simulation was presented. The analysis regards to propagation of wave crests and troughs within the transient and steady states. Achieved results will be used in the future for verification of data gathered during a laboratory experiment that is currently prepared by authors, where the acoustic wave is generated by a piezoelectric transducer.

\section{References}

[1] T. Boczar, D. Zmarzly, IEEE Trans. Diel. Electr. Insulat. 11, 433 (2004).

[2] F. Witos, Z. Gacek, Z. Opilski, Acta Phys. Pol. A 114, A-249 (2008). 
[3] T. Boczar, S. Borucki, A. Cichon, M. Lorenc, Acta Phys. Pol. A 114, A-21 (2008).

[4] F. Witos, Z. Gacek, P. Paduch, Mol. Quant. Acoust. 28, 271 (2007).

[5] T. Boczar, M. Szmechta, D. Zmarzły, Mol. Quant. Acoust. 28, 39 (2007).

[6] A. Szpakowski, C. Tyszkiewicz, T. Pustelny, Acta Phys. Pol. A 114, A-237 (2008).

[7] T. Boczar, S. Borucki, A. Cichon, Mol. Quant. Acoust. 28, 13 (2007).

[8] D. Wotzka, T. Boczar, D. Zmarzły, in: Conference Record of the 38 Winter School on Wave and Quantum Acoustics, Korbielów (Poland), Ed. A. Szpakowski, OG PTA, Gliwice 2009, p. 22.
[9] D. Wotzka, in: Conference Record of the IEEE 2nd International Students Conference on Electrodynamics and Mechatronics, Góra Św. Anny (Poland), Ed. T. Boczar, Technol. Univ. Opole, Opole 2009, p. 39.

[10] D. Wotzka, T. Boczar, D. Zmarzły, Acta Phys. Pol. A 116, 428 (2009).

[11] T. Boczar, S. Borucki, A. Cichon, M. Lorenc, Acta Phys. Pol. A 114, A-19 (2008).

[12] F. Witos, Z. Gacek, Acta Phys. Pol. A 116, 422 (2009). 